\title{
Improved preservation of human corneal basement membrane following freezing of donor tissue for epikeratophakia
}

\author{
Robert D Young, W John Armitage, Paul Bowerman, Stuart D Cook, David L Easty
}

\begin{abstract}
Current methods for the production of lenticules for epikeratophakia involve rapid freezing, cryolathing, and slow warming of the donor cornea. We have found that this procedure causes structural damage to the epithelial basement membrane in the donor cornea which may subsequently contribute to poor postoperative re-epithelialisation of the implant, leading to graft failure. Endeavouring to overcome these problems, the effects of cryoprotection of donor cornea were investigated, using dimethyl sulphoxide, in conjunction with different cooling and warming rates as part of the protocol for cryolathing. The structural integrity of the epithelial basement membrane zone (BMZ) was then assessed by electron microscopy and by immunofluorescence microscopy using antibodies to types IV and VII collagen, components of the basal lamina and anchoring fibrils respectively, and an antibody to a component of the anchoring filaments. No differences in the pattern of immunostaining for these components were detected, indicating that the composition of the BMZ was unaltered by the different treatment regimens applied. However, electron microscopy showed that preservation of basement membrane ultrastructure was markedly improved when cornea was warmed rapidly rather than slowly, both in cryoprotected and non-cryoprotected tissue. Epithelial cell retention and preservation of stromal architecture appeared superior in cryoprotected samples, while keratocyte structure was heterogeneous throughout the experimental groups. Further work is in progress to assess the efficacy of these protocols in the preservation of keratocyte viability in association with improved basement membrane structure in donor tissue for epikeratophakia.
\end{abstract}

(Br F Ophthalmol 1994; 78: 863-870)

Epikeratophakia was originally introduced in $1980^{\prime}$ as a reversible and simplified approach to corneal refractive surgery for aphakia, although it soon also found application in the surgical treatment of keratoconus ${ }^{2}$ and myopia. ${ }^{3}$ The technique involves reshaping a frozen disc of donor cornea on a cryolathe, to form a lenticule, which is then transplanted onto the de-epithelialised surface of Bowman's layer.

The results of epikeratophakia since its inception have traditionally been considered good, ${ }^{4-7}$ and while, in recent years, the popularity of the procedure may have declined in the United
States, good results continue to be achieved by the small number of British surgeons performing the technique. ${ }^{+}$However, no comprehensive account of its long term outcome has yet been published.

Several complications resulting in the failure of epikeratophakia have been reported, including infection, graft dehiscence, persistent interface haze or opacity, ulceration, and imperfect re-epithelialisation. Among these, the failure of host epithelial cells to migrate over and resurface the anterior face of the grafted tissue continues to be the major reason for the removal of epikeratophakia lenticules. ${ }^{8-10}$

Epithelial healing is itself a complex phenomenon involving mitosis of host cells at the graft periphery, centripetal migration, and attachment. In all of these processes the nature of the underlying substratum must be considered to be of profound importance, as shown by studies of cell growth and attachment in vivo and in vitro. ${ }^{11}{ }^{12}$ The integrity of the epithelial basement membrane in the cryolathed donor cornea, which becomes the presumptive substrate for host epithelial cells, may therefore be an important factor influencing the recovery of an intact and durable epithelial sheet over the newly grafted lenticule.

In preliminary observations both of cryolathed corneas prepared for epikeratophakia and of failed grafts, we identified several structural alterations to the epithelial basement membrane zone (BMZ), including disruption of the basal lamina and disorganisation of anchoring fibril complexes and anterior Bowman's layer. These changes appeared to be attributable to freezing rather than to the lathing procedure itself and were sufficiently extensive to warrant consideration as potential inhibitors of epithelial healing in the immediate postoperative period. The few studies carried out on the effects of freezing corneal tissue during the preparation of epikeratophakia lenticules have focused mainly on the viability of stromal keratocytes. ${ }^{1314}$ Factors such as cooling and warming rates and the presence of cryoprotective agents (for example, dimethyl sulphoxide), not only affect the survival of cells during freezing and thawing, but influence the location and quantity of ice, and thus the degree of structural disruption within tissues. ${ }^{15} \mathrm{We}$ therefore investigated these factors to determine whether the preservation of structural integrity of the anterior face of the prospective graft could be improved. The ultrastructural organisation of the $\mathrm{BMZ}$ was assessed by transmission electron microscopy and aspects of its composition were studied by immunofluorescence microscopy 
with monoclonal antibodies directed against specific components of the basal lamina and anchoring fibril structures.

\section{Materials and methods}

COOLING AND WARMING RATES

\section{Rapid cooling}

Samples were cooled at about $20^{\circ} \mathrm{C} / \mathrm{min}$ to $-50^{\circ} \mathrm{C}$ on the cryolathe. The tissue sample was attached by its epithelial surface to an aluminium arbour, which was clamped in the cryolathe chuck.

\section{Slow cooling}

Samples were cooled at $1^{\circ} \mathrm{C} / \mathrm{min}$ to $-50^{\circ} \mathrm{C}$ in a programmable controlled rate freezer (Planer Kryo 10-16).

\section{Rapid warming}

Frozen samples were immersed directly into medium at approximately $22^{\circ} \mathrm{C}$ (ambient temperature), which gave a warming rate of $>50^{\circ} \mathrm{C} / \mathrm{min}$

\section{Slow warming}

Frozen samples were allowed to thaw still attached to the cryolathe chuck, which gave a warming rate of $<20^{\circ} \mathrm{C} / \mathrm{min}$.

Corneas routinely processed for epikeratophakia were cooled and warmed on the cryolathe - that is, rapid cool/slow warm.

CRYOPROTECTANT ADDITION AND REMOVAL Samples were incubated in $10 \%$ (v/v) dimethyl sulphoxide (DMSO) in Eagle's minimal essential medium (MEM) for 15 minutes at $22^{\circ} \mathrm{C}$. The cryoprotectant was removed by dilution by transferring corneal tissue into $0.5 \mathrm{~mol} /$ litre sucrose in MEM at $22^{\circ} \mathrm{C}$. The sucrose acted as an osmotic buffer to limit the cell swelling that would have been induced by the reduction in DMSO concentration. ${ }^{16}$ After 15 minutes, the samples were placed into MEM alone.

\section{EXPERIMENTAL TREATMENTS}

The corneas were obtained within $23 \mathrm{~h}$ post mortem from a 60-year-old man, with no ocular disease, who had died from Hodgkin's lymphoma, which is a medical contraindication to transplantation of the corneas. Corneoscleral discs were excised and placed in Eagle's MEM. The corneas were then dissected free of sclera, cut into four equal quadrants, and subjected to the following treatments (summarised in the diagram):

Samples 1-4 were from the left cornea, and were not incubated in the cryoprotectant medium:

(1) processed for microscopy without further treatment;

(2) rapid cool, lathing to $0.35 \mathrm{~mm}$ thickness, slow warm (that is, similar to routine processing for epikeratophakia);
Treatment of tissue samples from paired human corneas
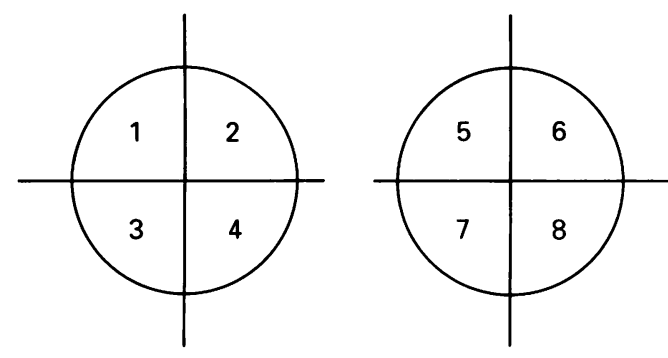

(3) rapid cool, slow warm (without lathing);

(4) rapid cool, rapid warm.

Samples 5-8 were from the right cornea, and were incubated in the cryoprotectant medium:

(5) addition and removal of cryoprotectant without freezing;

(6) slow cool, rapid warm with cryoprotectant;

(7) rapid cool, slow warm with cryoprotectant;

(8) rapid cool, rapid warm with cryoprotectant.

The samples were all processed for microscopy after these treatments.

IMMUNOFLUORESCENCE MICROSCOPY

Samples from each of the corneal sectors (1-8) were mounted in Tissue-tek medium on aluminium stubs and frozen by plunging into liquid nitrogen cooled isopentane. Frozen sections, $7 \mu \mathrm{m}$ thick, were cut on a Bright cryostat and mounted on slides coated with Biobond adhesive. Sections were exposed to monoclonal antibodies to type IV collagen (goat anti-human), a component of the basal lamina; type VII collagen (LH7.2, mouse anti-human), a component of the anchoring fibrils; or to an antibody to a component of the anchoring filaments (LH39, mouse anti-human). Type IV antibody was an affinity purified reagent supplied by Southern Biotechnology Associates. LH7.2 and LH39 were generously donated by Professor I Leigh, Department of Experimental Dermatology, London Hospital Medical College. After $2 \mathrm{~h}$ incubations in the primary monoclonal antibody and washing in $1 \%$ bovine serum albumin (BSA) in phosphate buffered saline (PBS), or in PBS/BSA alone in the case of control preparations, the sections were incubated with secondary antibodies conjugated to fluorescein isothiocyanate (FITC), followed by further washing and mounting in Citifluor. They were then viewed using a Leitz microscope equipped for FITC fluorescence and phase optics.

\section{ELECTRON MICROSCOPY}

Samples from each treated corneal sector were fixed at room temperature for $2-3 \mathrm{~h}$ in $2.5 \%$ glutaraldehyde in $0.1 \mathrm{M}$ sodium cacodylate buffer containing $4 \mathrm{mM}$ calcium chloride at $\mathrm{pH}$ $7 \cdot 2$. After rinsing in buffer, they were post-fixed in buffered $1 \%$ osmium tetroxide, stained en bloc in $0.5 \%$ aqueous uranyl acetate and dehydrated in an ethanol series. Sections were prepared from the Araldite embedded samples, 

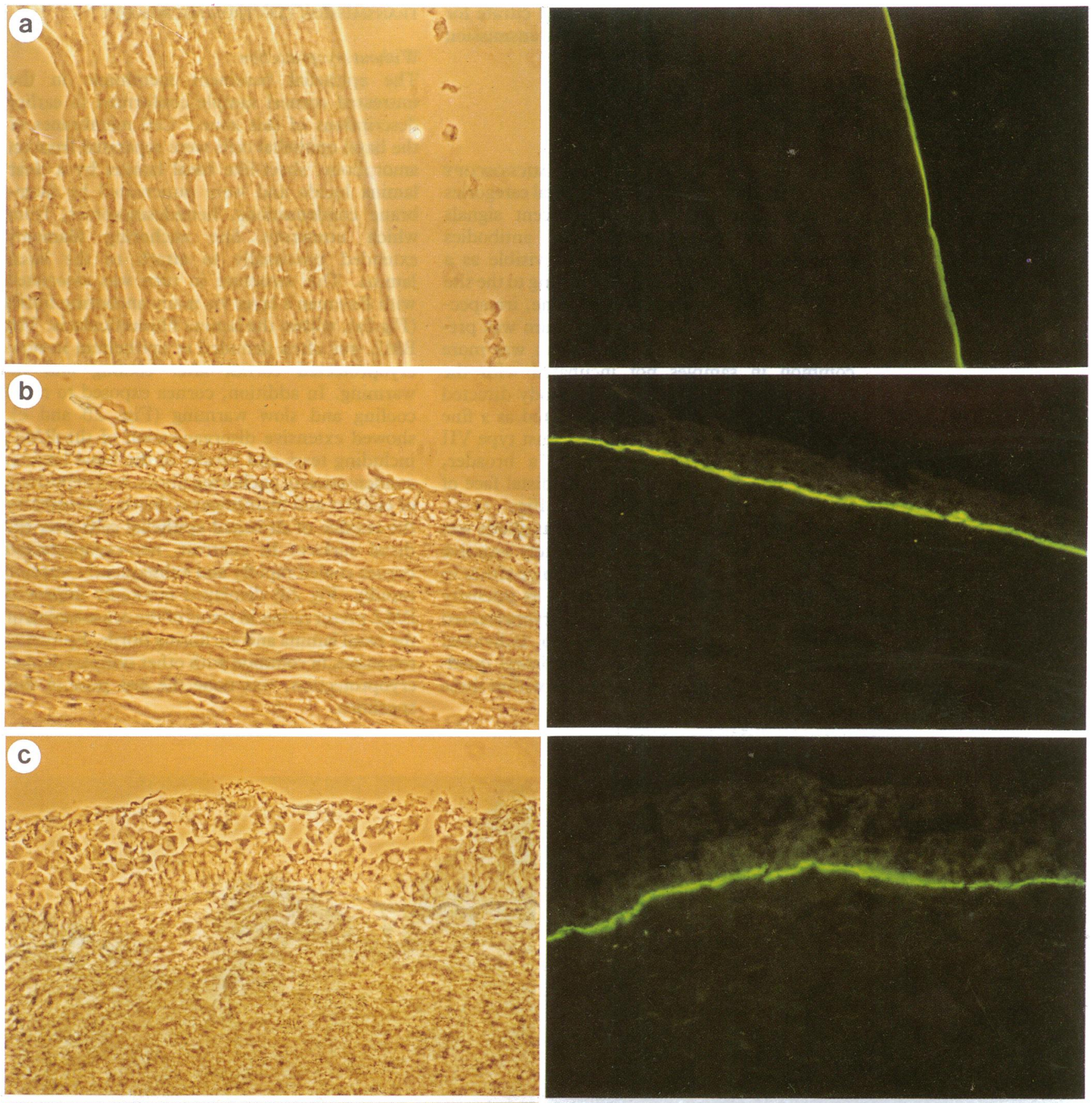

d
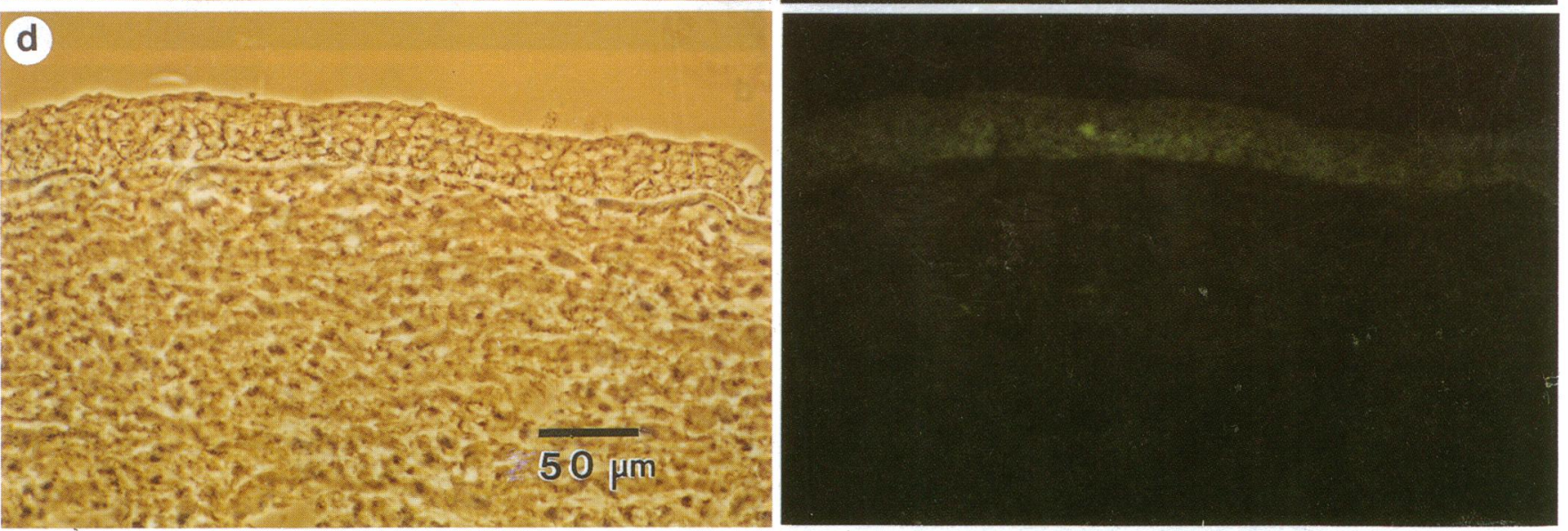

Figure 1 Immunolocalisation of human corneal basement membrane zone components. Examples of human cornea exposed to different procedures in relation to cryoprotection and rates of cooling and warming. Comparative phase (left) and fluorescence (right) microscopy images show (a) cornea, without cryoprotection (sample 4), exposed to rapid cool and rapid warm, stained for type IV collagen; (b) cryoprotected cornea, exposed to rapid cool and rapid warm (sample 8), stained for type VII collagen; (c) cryoprotected cornea, exposed to rapid cool and slow warm (sample 7), stained with LH39 for anchoring filaments; (d) control preparation: cryoprotected cornea, exposed to rapid cool and rapid warm (sample 8), incubated without primary antibody. 
contrasted in uranyl acetate and lead citrate for examination in a Philips 400 transmission electron microscope at $80 \mathrm{kV}$.

\section{Observations}

PHASE AND IMMUNOFLUORESCENCE MICROSCOPY Corneal sections from all experimental categories revealed strongly positive fluorescent signals towards all three monoclonal antibodies employed. Localised staining was visible as a continuous linear signal corresponding to the site of the epithelial basement membrane, irrespective of whether or not the epithelium was preserved intact. Epithelial detachment was more common in samples not incubated with the cryoprotectant (Fig la). The antibody directed against type IV collagen was detected as a fine line of fluorescence, while that against type VII collagen and LH39 appeared as a broader, intense signal coincident with the distal face of Bowman's layer (for example, Figs la-c). Control sections where the primary antibody was omitted, in each case were completely negative (Fig 1d). There was no clear difference either in the distribution of the signal or in its intensity between cryoprotected or non-cryoprotected corneal samples. In addition, no individual differences could be assigned to corneal quadrants receiving different treatments within these two groups.

\section{TRANSMISSION ELECTRON MICROSCOPY}

\section{Without cryoprotection}

The epithelial basement membrane in the untreated corneal sample conformed to earlier descriptions of this zone. ${ }^{17}$ Cell membranes of the basal epithelial cells were separated from an amorphous, sheet-like basal lamina by a clear lamina lucida and were punctuated by membrane condensations, hemidesmosomes, from which numerous fine anchoring filaments extended proximally to attach to the basal lamina. This structure in turn was associated with Bowman's layer via many anchoring fibrils (Fig 2a), within the lamina fibroreticularis. The BMZ was often denuded of epithelium in noncryoprotected cornea after cooling and rewarming. In addition, cornea exposed to rapid cooling and slow warming (Figs $2 \mathrm{~b}$ and $\mathrm{c}$ ), showed extensive disorganisation of the $\mathrm{BMZ}$, including tears and breaks in the basal lamina, condensation of anchoring fibrils, and deposition of cellular and membranous debris, presumably the residue of damaged epithelial cells over the distal surface. In contrast, non-cryoprotected cornea cooled rapidly and subsequently warmed rapidly (Fig 2d) displayed markedly improved $\mathrm{BMZ}$ preservation in terms of the organisation of the basal lamina and anchoring fibrils. Epithelial retention, in common with other samples denied cryoprotection, was none the less poor in this specimen.
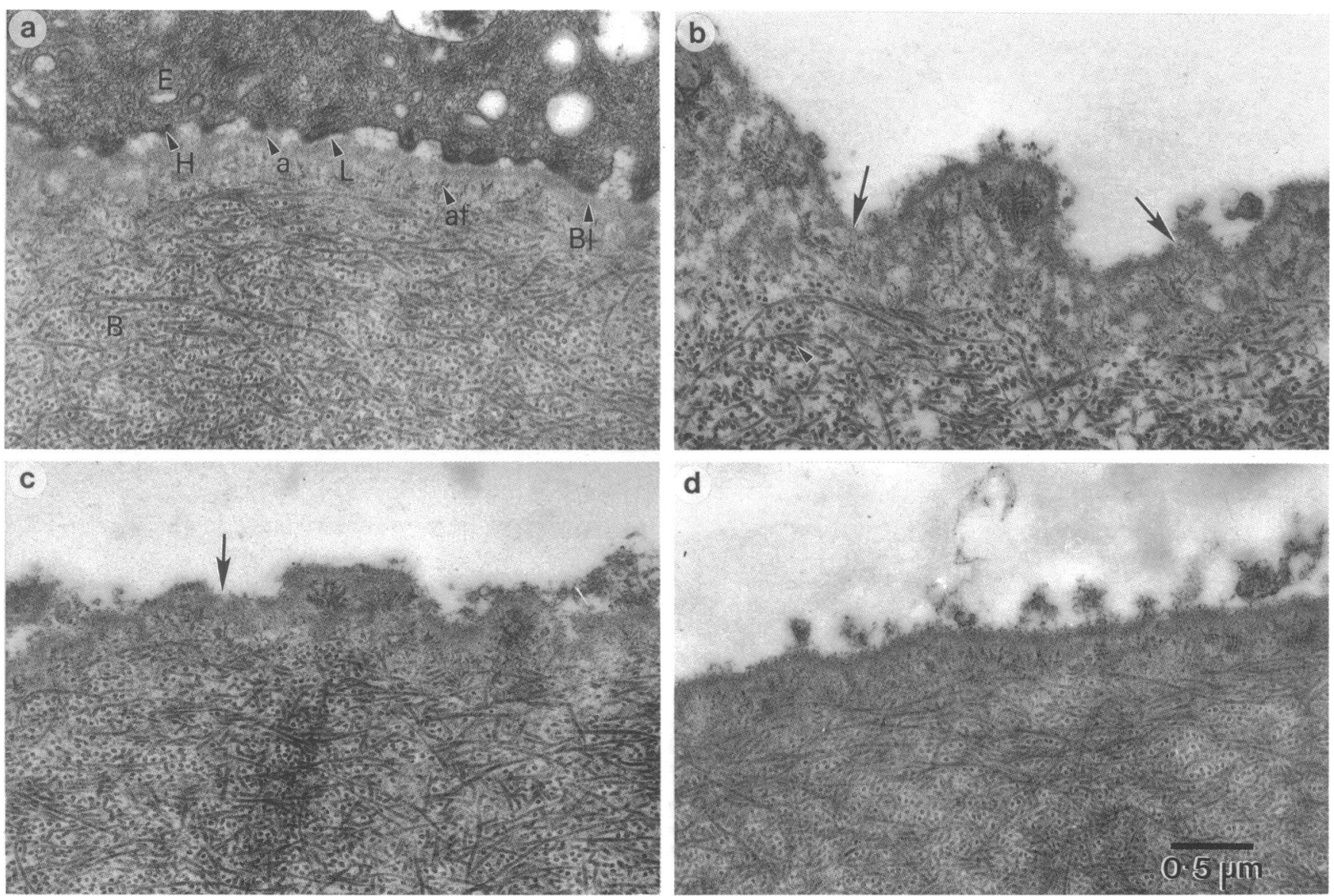

Figure 2 Electron microscopy of basement membrane zone (BMZ) in human cornea without cryoprotection, treated as follows: (a) no cooling (sample 1);

(b) rapid cool, cryolathed, and slow warm (sample 2); and (c) rapid cool and slow warm (sample 3), showing loss of epithelium, breaks in basal lamina (arrows) and condensation of anchoring fibrils. (d) Rapid cool and rapid warm (sample 4), showing improved BMZ preservation. E, epithelium; Bl basal lamina; $H$, hemidesmosomes; $L$, lamina lucida; $a$, anchoring filaments; af, anchoring fibrils; $B$, Bowman's layer. 

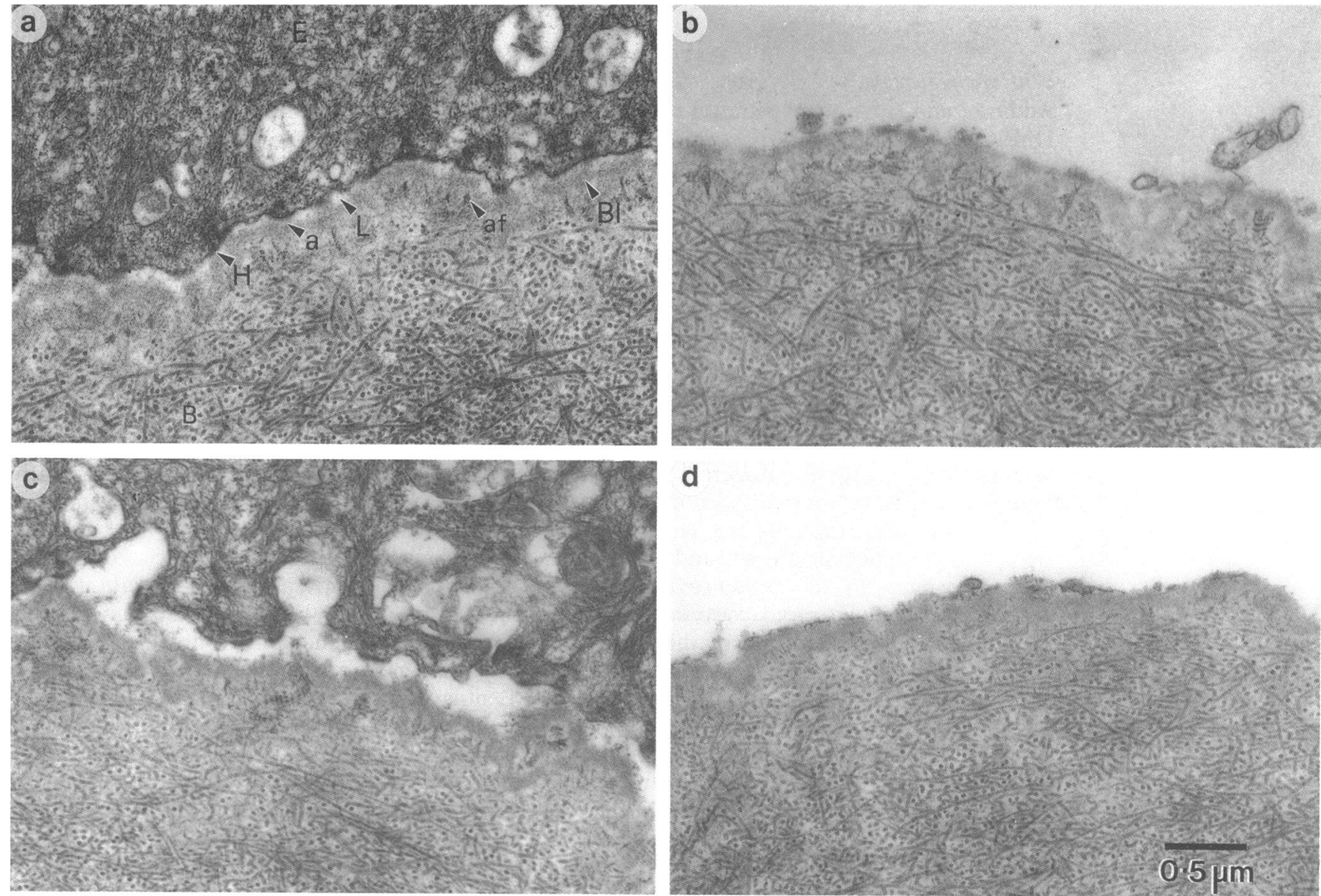

d

Figure 3 Electron microscopy of basement membrane zone $(B M Z)$ in human cormea with cryoprotection, treated as follows: (a) no cooling (sample 5): cryoprotection causes no obvious structural alteration to the $B M Z ;(b)$ slow controlled cool and rapid warm in medium (sample 6): diffuse and interrupted appearance of basal lamina; (c) rapid cool and slow warm (sample 7); (d) rapid cool and rapid warm in medium (sample 8): optimal preservation of BMZ. $E$, epithelium; $B l$, basal lamina; $H$, hemidesmosomes; $L$, lamina lucida; $a$, anchoring filaments; af, anchoring fibrils; $B$, Bowman's layer.
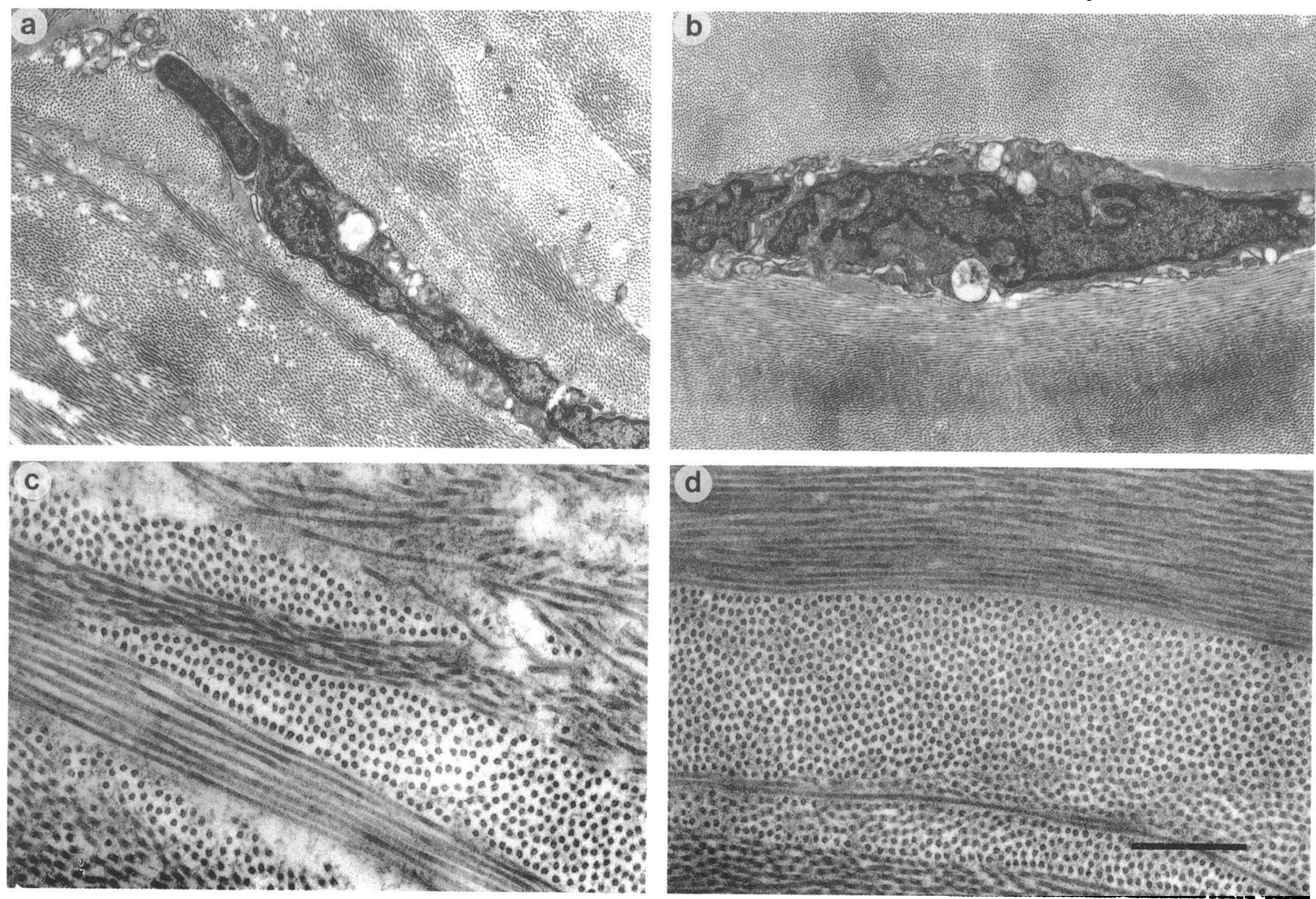

Figure 4 Electron microscopy of human corneal keratocytes and stroma. Stromal keratocytes (a) in non-cryoprotected cornea, after rapid cool, cryolathing and slow warm (sample 2); and (b) in cryoprotected cornea after rapid cool and rapid warm (sample 8), showing nuclear crenation, vacuolation and rupture of cellular organelles. Stromal lamellae are disorganised with increased collagen interfibrillar distance and intralamellar lakes in (c) non-crvoprotected cornea after rapid cool, cryolathing, and slow warm (sample 2), while in cryoprotected cornea $(d)$, after rapid cool and rapid warm (sample 8), stromal integrity is well preserved.
$B a r=1.5 \mu m(a)$ and $(b) ; 0.5 \mu m,(c)$ and $(d)$. 
With cryoprotection

Addition and subsequent removal of $10 \%(\mathrm{v} / \mathrm{v})$ DMSO without freezing appeared to cause no structural alteration to the BMZ (Fig 3a). The organisation of anchoring fibrils and filaments in association with the basal lamina and hemidesmosomes was comparable with that seen in cornea not exposed to the cryoprotectant (Fig 2a). Epithelial retention in cryoprotected corneal samples was found to be generally better than in corneas frozen without cryoprotectant, although some sites were located where loss or separation of epithelium was evident (Figs $3 b$ and $3 d$ ). In cryoprotected cornea, cooled rapidly and warmed slowly (Fig 3c), distortion of the basal lamina was observed, reminiscent of that seen in $B M Z$ in samples frozen in the same way but without cryoprotectant (Figs $2 \mathrm{~b}$ and c).

Slow cooling in the presence of cryoprotectant, followed by rapid warming (Fig 3b), reduced the gross distortion of the $B M Z$ and avoided the changes in the anchoring fibril component that were seen in cornea cooled rapidly and warmed slowly (Figs 2b, 2c, 3c). However, in this case the basal lamina appeared diffuse and interrupted, presumably in response to the deposition of large ice crystals during slow cooling. The most favourable preservation of $\mathrm{BMZ}$ structure was identified in cryoprotected cornea subjected to a regimen of rapid cooling and rapid warming (Fig 3d). Here, all components of the $\mathrm{BMZ}$ appeared intact although at sites of epithelial detachment, a fine deposit of basal cell membrane, including hemidesmosomes and anchoring filaments, could be observed attached to the basal lamina (Fig 3d).

\section{Keratocytes and stroma}

A wide range of morphological changes were displayed by keratocytes in response to different cooling and warming procedures, both with or without cryoprotection. Cytoplasmic vacuolation, disruption of cell membrane and organelles, together with nuclear crenation and aggregation of nuclear chromatin could be located in cells of all categories (for example, Figs $4 a$ and b). Isolated cells with comparatively normal ultrastructural features were also present.

Observations on collagen fibrils in stromal lamellae were made in all eight experimental categories. In general, rapid cooling and rapid warming of cryoprotected stroma gave rise to less disruption of lamellae and of the component fibrillar organisation, than that produced by rapid cooling and slow warming with cryoprotection (for example, Figs $4 c$ and d).

\section{Discussion}

The major clinical complications associated with epikeratophakia have been related to the failure of host epithelial cells to migrate over and establish a normal epithelium on the graft surface. ${ }^{8-10}$ Even when the epithelium is reestablished, specular microscopy has shown that there may still be abnormalities in cell shape and organisation which may persist up to 16 months after surgery. ${ }^{18}$ Those aspects of the epikeratophakia procedure that are responsible for poor re-epithelialisation remain obscure; but epithelial complications are not common with penetrating or lamellar keratoplasty. Problems with both host and donor tissue, such as manipulation of the recipient cornea or differences in viability of the graft tissue, have been put forward in explanation for epithelial insufficiency. ${ }^{8}$ The steepened corneal topography at the host-graft interface, which may impede cell migration and expose loosely attached cells to the action of the eyelids has also been considered. Steps in the preparation of lenticules, including freezing, lathing, lyophilisation, and the use of the corneal press have all been implicated in causing structural damage to the lenticule which might inhibit the establishment of a normal functional epithelium. ${ }^{8}$

Epithelial healing is closely associated with the renewal of the $\mathrm{BMZ}$, a complex structural interface between the basal cells and underlying Bowman's layer, including components mediating attachment of the cells to their substratum. Lenticules removed postoperatively owing to persistent epithelial defects exhibited a marked reduction in numbers of cell attachment structures, hemidesmosomes, and basal lamina. ${ }^{19}$ Factors influencing the deposition of a functional basal lamina and the reformation of hemidesmosomes in healing epithelia have been little studied. However, the adhesion, migration, and mitosis of epithelial cells are known to be enhanced by basement membrane components, including type IV collagen. ${ }^{12}$ The elegant studies of Gipson $e^{2} a l^{20}$ on $\mathrm{BMZ}$ renewal in recombined rabbit corneal epithelial sheets and denuded stroma in vitro showed that the sites of residual anchoring fibril insertions into the basal lamina may represent nucleation sites for the formation of new hemidesmosomal attachments in the healing epithelial cells. However, subsequent studies on the sequence of reassembly of the BMZ under different experimental conditions have not found universal agreement. ${ }^{2122}$ Hemidesmosomes may precede anchoring fibril formation in situations where the basal lamina and superficial stroma are absent or damaged, ${ }^{21}$ and the presence of type VII collagen may not be essential for hemidesmosome formation per se because sheep with the disease dystrophic epidermolysis bullosa, where type VII collagen is not expressed, have intact hemidesmosomes.

The potential for epithelial cells to attach and restore a surface layer, even on suboptimal substrates is clearly demonstrated by these previous studies. It seems reasonable to expect the process to be greatly facilitated by the provision of an intact undamaged basal lamina for new epithelial cell growth. Yet the effects of freezing on structural integrity of epikeratophakia lenticules have not previously been assessed in detail. We found that current methods of cryolathing involving rapid cooling followed by slow warming, without cryoprotection, gave rise to considerable distortion of the $\mathrm{BMZ}$, with tears in the basal lamina and condensation of anchoring fibrils. Fine structural damage to the surface of the lenticule can therefore be attributed specifically to the freezing and warming procedures rather than the lathing process itself. These modifications to $\mathrm{BMZ}$ fine structure may 
be important contributory factors in the inhibition of epithelial attachment and re-surfacing.

Our studies show that the integrity of the graft surface can be markedly improved by rapid rather than slow warming of frozen cornea. This was achieved by freezing the cornea in situ on the cryolathe and then quickly transferring the frozen cornea to tissue culture medium at $22^{\circ} \mathrm{C}$. Rapid warming would have allowed less time for structural disruption through recrystallisation of ice, the process whereby larger ice crystals grow at the expense of smaller, and thus less thermodynamically stable, crystals. In addition, the presence of antibody binding to type IV and type VII collagen and to anchoring filaments demonstrates the compositional integrity of basal lamina, anchoring fibrils, and anchoring filaments following this treatment. Type IV and type VII collagens in the basal lamina and anchoring fibrils, together with laminin, nidogen, and heparan sulphate proteoglycan in the lamina lucida of the $\mathrm{BMZ}$ have all been identified as potential ligands for the attachment of basal epithelial cells. "Immunogold electron microscopy demonstrated that the anchoring filament antibody used in this study showed specificity towards sites in the lamina lucida below hemidesmosomes, corresponding to the anchoring filaments. ${ }^{23}$ Although the precise epitope for this antibody is as yet unknown, it may be related to epiligrin, a recently discovered glycoprotein which appears to be an important cell adhesion ligand for epidermal and other epithelial cells via the integrin $\alpha 3 \beta 1$ expressed on their cell membranes. ${ }^{24}$

The improvement in structure of the BMZ brought about by rapid warming was observed irrespective of the presence of cryoprotective agent. On the other hand, retention of epithelium and integrity of stromal lamellae did appear to be better when corneal tissue was frozen in the presence of DMSO. Such a result would not be unexpected since not only would the DMSO protect the epithelial cells against freezing injury, but less ice would have formed in the stroma owing to the colligative effect of the presence of the cryoprotectant. These aspects warrant further investigation because cryoprotection of tissue for refractive keratoplasty has previously been discouraged, mainly as a result of the putative deleterious effects of glycerol on endothelial cells in the recipient cornea. ${ }^{25}$ The original purpose of cryoprotection, to facilitate the survival of keratocytes during freezing, was questioned because viability of donor keratocytes was considered not to be essential for the survival of the graft, although they could be important in relation to stromal integrity. Moreover, keratocyte epithelial cell interactions are now thought to be important in the homeostasis of the BMZ. ${ }^{26}$ The death of keratocytes from freeze injury during the preparation of lenticules may result in the absence of viable cells from the graft stroma for months after surgery, before slow repopulation takes place by cell migration from the recipient cornea. ${ }^{1327}$ Cryoprotective agents would be expected to facilitate keratocyte survival as demonstrated recently. ${ }^{28}$ In our study, however, keratocyte structure was heterogeneous in all groups and the effects of cryopro- tection on keratocyte viability have yet to be determined. Previous authors have reported improved keratocyte viability following slow cooling in the absence of cryoprotection, ${ }^{29}$ yet our studies showed a slow cooling rate to be detrimental to the preservation of the basal lamina. Used in combination with rapid cooling and warming rates, which we found favour $B M Z$ preservation, cryoprotective agents may permit the retention both of surface morphology and keratocyte viability in the prospective graft. Further work is in progress to assess the efficacy of these protocols for the improvement of graft tissue for epikeratophakia in order to overcome problems of re-epithelialisation.

This work was supported by a project grant from the Guide Dogs for the Blind Association of the United Kingdom. The authors are indebted to Professor I M Leigh for generously donating monoclonal antibodies against $\mathrm{BMZ}$ components and to Mrs A C Phillips for skilled assistance with cryostat sectioning and fluorescence microscopy.

1 Kaufman HE. The correction of aphakia. Am $\mathcal{F}$ Ophthalmol 1980;89: $1-10$.

2 Kaufman HE, Werblin TP. Epikeratophakia for the treatment of keratoconus. Am $\mathcal{F}$ Ophthalmol 1982; 93: 342-7.

3 McDonald MB, Klyce SD, Suarez H, Kandarakis A Friedlander MH, Kaufman HE. Epikeratophakia for myopia correction. Ophthalmology 1985; 92: 1417-22.

4 McDonald MB, Kaufman HE, Aquavella JV, Durrie DS Hites DA, Hunkeler ID, et al. The nationwide study of epikeratophakia for aphakia in adults. Am $\mathcal{f}$ Ophthalmo 1987; 103: 358-65.

5 McDonald MB, Kaufman HE, Aquavella JV, Durrie DS, Hites DA, Hunkeler ID, et al. The nationwide study of epikeratophakia for myopia. Am $\mathcal{F}$ Ophthalmol 1987; 103: 375-83.

6 McDonald MB, Kaufman HE, Durrie DS. Epikeratophakia for keratoconus, the nationwide study. Arch Ophthalmol 1986; 104: 1294-300.

7 Halliday BL. Epikeratophakia for aphakia, keratoconus and myopia. Brf Ophthalmol 1990; 74: 67-72.

8 Binder PS, Zavala EY. Why do some epikeratoplasties fail? Arch Ophthalmol 1987; 105: 63-9.

9 Binder PS, Baumgartner SD, Fogle JA. Histopathology of a case of epikeratophakia (aphakic epikeratoplasty). Arch Ophthalmol 1985; 103: 1357-63.

10 Rodrigues $M$, Nirankari V, Rajagopalan S, Jones $K$, Funderburgh J. Clinical and histopathologic changes in the host cornea after epikeratoplasty for keratoconus. Am host cornea after epikeratoplasty

11 Yurchenco PD, Schittny JC. Molecular architecture of basement membranes. FASEB B 1990; 4: 1577-90.

12 Olivero DK, Furcht LT. Type IV collagen, laminin and fibronectin promote adhesion and migration of rabbit lens epithelial cells in vitro. Invest Ophthalmol Vis Sci 1993; 34 2825-34.

13 Rich LF, Friedlander MH, Kaufman HE, Granet N. Keratocyte survival in keratophakia lenticules. Arch Ophthalmol 1981; 99: 677-80.

14 Lee TJ, Wan WL, Kash RL, Kratz KL, Schanzlin DJ. Keratocyte survival following a controlled-rate freeze. Invest Ophthalmol Vis Sci 1985; 26: 1210-5.

15 Mazur P. Freezing of living cells: mechanisms and implications. Am f Physiol 1984; 247: c125-42.

16 Leibo SP, Mazur P. Methods for the preservation of mammalian embryos by freezing. In: Daniel JC, ed. Methods of mammalian reproduction. New York: Academic Press, 1978: 179-201.

17 Gipson IK, Spurr-Michaud SJ, Tisdale SJ. Anchoring fibrils form a complex network in human and rabbit cornea. Inves Ophthalmol Vis Sci 1987; 28: 212-20.

18 Rao GN, Grant S, Aquavella JV. Specular microscopy of the corneal epithelium after epikeratophakia. Am $\mathcal{F}$ Ophthalmol 1987; 103: 392-6.

19 Azar DT, Spurr-Michaud SJ, Tisdale AS, Moore MB, Gipson IK. Reassembly of the corneal epithelial adhesion structures following human epikeratoplasty. Arch Ophthalmol 1991; 109: 1279-84

20 Gipson IK, Grill SM, Spurr SJ, Brennan SJ. Hemidesmosome formation in vitro. F Cell Biol 1983; 97: 849-57.

21 Stock EL, Kurpakus MA, Sabol B, Jones JC. Adhesion complex formation after small keratectomy wounds in the cornea. Invest Ophthalmol Vis Sci 1992; 33: 304-13.

22 Gipson IK, Spurr-Michaud SJ, Tisdale SJ, Keough M Reassembly of the anchoring structures of the corneal epithelium during wound repair in the rabbit. Invest Ophthalmol Vis Sci 1989; 30: 425-34.

23 Almeida BM, Challacombe SJ, Eveson JW, Smith CG, Leigh IM. A novel lamina lucida component of epithelial and clonal antibody. F Pathol 1992; 166: 243-53.

24 Carter WG, Ryan MC, Gahr MJ. Epiligrin, a new cell adhesion ligand for integrin $\alpha 3 \beta 1$ in epithelial basement membrane. Cell 1991; 65: 599-610. 
25 Binder PS, Zavala EY, Deg J, Akers PH. Refractive keratoplasty. Tissue dyes and cryoprotective solutions. Arch plasty. Tissue dyes and cryop
Ophthalmol 1983; 101: 1591-6.

26 Marinkovich MP, Keene DR, Rimberg CS, Burgeson RE Cellular origin of the dermal-epidermal basement membrane. Developmental Dynamics 1993; 197: 255-67.

27 Binder PS, Beale JP, Zavala EY. The histopathology of a case of keratophakia. Arch Ophthalmol 1982; 100: 101-8. 28 Kratz-Owens KL, Huff JW, Schanzlin DJ. New cryoprotectant for cryorefractive surgery. $\mathcal{F}$ Cataract Refract Surg

29 Lee TJ, Wan WL, Kash RL, Kratz KL, Schanzlin DJ. Keratocyte survival following a controlled-rate freeze. Invest Ophthalmol Vis Sci 1985; 26: 1210-5. 